\title{
AVALIAÇÃO DO RISCO DE QUEDA EM IDOSOS INDEPENDENTES
}

\author{
Fábio Augusto da Silva, Maria Isabel Borges de Matos, Larissa Sapucaia Ferreira Esteves
}

Universidade do Oeste Paulista - UNOESTE, Curso de Enfermagem, Presidente Prudente, SP. e-mail: fabioaugusto010101@hotmail.com

\section{RESUMO}

O presente estudo tem por objetivo avaliar o risco de quedas em idosos autônomos residente em um Programa de Habitação Municipal. A metodologia utilizada foi de pesquisa quantitativa, não experimental, de natureza descritiva e transversal. Foi realizada em uma vila de idosos na cidade de Presidente Prudente, com 26 idosos. Os dados foram obtidos por meio de um questionário sócio demográfico e por meio da aplicação de dois instrumentos. Os dados quantitativos foram analisados por meio da estatística simples e descritiva. Observamos que prevaleceram idosos do sexo masculino, 65,3\% possuem mais de 70 anos, o tempo médio de estudo foi de 2,8 anos. Avaliação cognitiva, 46,2\% apresentou baixo desempenho, $65,4 \%$ dos entrevistados apresentou baixo risco de quedas. Como conclusão, acredita-se que ações interdisciplinares e intersetoriais sejam necessárias para garantir a funcionalidade física e cognitiva da população.

Palavras-chave: idoso, idoso fragilizado, enfermagem, fatores de risco, queda.

\section{FALL RISK ASSESSMENT IN INDEPENDENT ELDERLY}

\section{ABSTRACT}

The present study aims to evaluate the risk of falls in autonomous elderly domiciled in a Municipal Housing Program. The methodology applied was quantitative research, non-experimental, of descriptive and crosssectional nature. It was conducted in an elderly inhabitant in Presidente Prudente, with 26 elderly people. The data has been obtained by a social-demographic questionnaire and by two instruments implementation. Quantitative data were analyzed through simple and descriptive statistics. It was observed that male elderly prevail, $65.3 \%$ are over 70 years old. The mean time of study was of 2.8 years. The cognitive evaluation $46.2 \%$ presented low performance, $65.4 \%$ of interviewed presented low risk of falls. Thus, in conclusion, it is believed that interdisciplinary and intersectoral actions may need to guarantee physical and cogntive function of population.

Keywords: elderly, elderly, frail, nursing, risk factors, fall.

\section{INTRODUÇÃO}

A queda é a principal causa de eventos traumáticos em idosos representando $90 \%$ dos atendimentos hospitalares desta população. Ao analisar os momentos em que esse evento aconteceu na população idosa, o autor ${ }^{1}$ revela que $79,6 \%$ sofreram queda da própria altura e $4 \%$ sofreram quedas em ônibus. Como principais complicações, destacam-se $22,4 \%$ sofreram lesões de superfícies externas, 15,1\% traumatismo crânio encefálico leve, $13,1 \%$ teve traumas de membros inferiores e 10,5\% da população estudada teve fratura de fêmur².
Os fatores que podem levar o idoso a queda estão relacionados tanto por causas intrínsecas quanto por extrínsecas. Os fatores intrínsecos estão diretamente ligados a alterações fisiológicas e patológicas como a idade, doenças crônicas, polifarmácia, depressão, diminuição da cognição, diminuição da capacidade funcional entre outras. Já os fatores extrínsecos estão diretamente ligados ao ambiente externo, como iluminação, mobília e estrutura física da casa ou lugar que frequenta constantemente ${ }^{3}$.

Várias são as escalas que podem auxiliar na avaliação do risco de quedas em idosos. Em 
2003, Gomes e colaboradores realizaram a tradução e adaptação para a língua portuguesa de uma escala para avaliar o equilíbrio e marcha, e consequentemente o risco de queda, a escala é denominada Performance Oriented Mobility Assessment ou Escala de Avaliação de Equilíbrio e Marcha de Tinneti ${ }^{4}$.

A escala de Avaliação de Equilíbrio e Marcha de Tinneti foi elaborada com a partir da análise dos seguintes aspectos: da marcha como a velocidade, distância do passo, simetria do equilíbrio em pé, girar e também mudanças com os olhos fechados. A pontuação varia de 0 a 28 , sendo que a contagem para cada exercício varia de 0 a 1 ou de 0 a 2 pontos, a pontuação menor que 19 indica risco 5 vezes maior de quedas ${ }^{2}$.

Sendo assim, a pergunta principal que mobilizou esta pesquisa foi: quais são os índices de risco de queda de idosos que vivem sozinhos em uma vila específica para essa população? Idosos que vivem sozinhos em comunidades podem apresentar riscos significativos para quedas?

Os objetivos desse estudo foram caracterizar a população idosa que reside em um Programa Vila da Dignidade, avaliar o risco de quedas, identificar os fatores que podem estar relacionados com os índices encontrados $\mathrm{e}$ apresentar medidas que podem ser tomadas para evitar as futuras quedas.

\section{METODOLOGIA}

Trata-se de uma pesquisa quantitativa, não experimental, de natureza descritiva e transversal realizada em uma vila de idosos de uma cidade do interior paulista. A pesquisa foi realizada em uma vila construída pela prefeitura municipal da cidade em parceria com o governo do Estado de São Paulo inaugurada em dezembro de 2010 que faz parte do Programa Vila da Dignidade (Decreto Estadual no. 56.448/2010). O conjunto habitacional, composto por 24 casas, é específico para pessoas idosas. Para residir nesta vila, os idosos devem possuir autonomia para as tarefas diárias, ter renda mensal de até dois salários mínimos, morar sozinhos há mais de dois anos na mesma cidade e vivenciar situação de vulnerabilidade social. As residências foram planejadas de acordo com parâmetros específicos que garantem facilidades principalmente aos indivíduos com dificuldade de locomoção, temporária ou permanente, acessibilidade e segurança ${ }^{4}$.
Participaram desse estudo 26 idosos que residem no Programa Vila da Dignidade de um município do interior paulista. Cada idoso reside sozinho em sua casa, com exceção de um casal que mora no mesmo domicílio. Os dados foram obtidos por meio de um questionário sócio demográfico elaborado pelos próprios autores e por meio da aplicação de dois instrumentos testados, validados e indicados pelo Ministério da Saúde para avaliação de idosos: o Mini Exame do Estado Mental $^{3}$ e o Escala de avaliação do equilíbrio e marcha de Tinneti ${ }^{2}$. Ambos instrumentos podem ser aplicados por qualquer profissional de saúde, pois trata-se de instrumentos de caráter multiprofissional e que compõe o arsenal de testes da Avaliação Global do Idoso proposto pelo Ministério da Saúde, publicado em $2006^{2}$.

Para a coleta de dados os pesquisadores reuniram os moradores com auxílio do Agente Comunitário de Saúde da Estratégia de Saúde da Família do bairro no próprio salão comunitário da vila. Neste dia explicaram os objetivos da pesquisa e apresentaram o instrumento de coleta de dados especificando cada item a ser obtido respeitando o tempo de compreensão de cada sujeito. Na sequência, o Termo de Consentimento Livre e Esclarecido foi explicado e após obter as assinaturas, agendaram a melhor data e hora para cada idoso que aceitou participar do estudo. O trabalho foi aprovado pelo Comitê de Ética em Pesquisa da Universidade Oeste Paulista (CAEE: 45459515.7.0000.5515), respeitando todas as diretrizes da Resolução 466/13 do Conselho Nacional de Saúde.

Para organização dos dados, foi elaborado uma planilha no programa Excel ${ }^{\circ}$, sendo alimentada diariamente após a coleta. Para a identificação da relação das escalas Tinnet e MEEM com as demais variáveis coletadas pelo instrumento, utilizou-se a técnica nãoparamétrica conhecida como Coeficiente de Correlação de Spearman, essa técnica indica a existência da relação, sendo considerada a indicação de existência o nível de significância estatística de $p<0,05^{6,7}$.

\section{RESULTADOS}

Dos 26 idosos entrevistados $53,8 \%$ são do sexo masculino e $65,3 \%$ possuem mais de 70 anos, sendo a media de idade de $72 \pm 6,68$ anos. Em relação à escolaridade, trata-se de uma população semi-analfabeta, considerando que $100 \%$ dos respondentes estudaram até no 
máximo o ensino fundamental, sendo a média dos anos de estudo 2,8 $\pm 1,84$ anos. Quando mensurados a quantidade de comorbidades prevalentes na população estudada, observou que há em média duas doenças crônicas por idoso. Em relação ao tipo de doenças crônicas, os agravos prevalentes são Hipertensão Arterial Sistêmica (HAS) e Déficit visual, com 34,6\% e $30,8 \%$, respectivamente.

Um dado em especial que chamou atenção é o cuidado com a saúde, pois $88,5 \%$ dos idosos relataram que procuram a Estratégia de Saúde da Família (ESF) com frequência, indicando que estes estão acompanhados pela equipe de saúde do bairro. Dos 26 idosos que participaram do estudo, $61,5 \%$ participam de grupos oferecidos pela ESF. Por meio de tal fato é possível afirmar que boa parte deles demonstra ter comportamentos positivos em relação à sua saúde, além de estar engajado em grupos de convívio onde amplia as possibilidades de melhorar suas relações interpessoais e social ${ }^{9}$.

Após análise do estado cognitivo (aplicação do MEEM) foi possível evidenciar que $46,2 \%$ da população pesquisada apresentou baixo desempenho cognitivo (pontuação igual ou inferior a 23 pontos). Cabe ressaltar que para o cálculo foi considerado o nível de escolaridade como orienta os autores do instrumento.

A análise de regressão estatística mostrou que a escolaridade foi uma das variáveis que obtiveram significância estatística $(p<0,05)$ em relação à pontuação do $\mathrm{MEEM}^{5}$ identificado, ou seja, a escolaridade obteve $p=0,0357$, evidenciando que a escolaridade foi um fator significante para os achados da avaliação cognitiva.

No que tange o risco de quedas, $65,4 \%$ dos idosos entrevistados possuem baixo risco para quedas e $34,6 \%$ apresentaram risco moderado para queda. A média da pontuação na escala de Tinneti ${ }^{2}$ foi de $24,8 \pm 2,19$ pontos. A pontuação total máxima que uma pessoa idosa pode atingir na Avaliação do Risco de Quedas é de 28 pontos e quanto menor a pontuação maior o problema ${ }^{2}$. Não foi encontrado idoso com alto risco para quedas dentre os sujeitos avaliados.

As variáveis que obtiveram significância estatística para esse resultado foram: idade e a presença de algumas doenças crônicas tais como cardiopatias, distúrbios urinários, osteoporose e sequelas resultantes de Acidentes Vasculares Encefálicos. Não houve significância estatística entre os escores do MEEM com os escores obtidos por meio da Escala de Avaliação do Equilíbrio e da Marcha de Tinneti ${ }^{2}$. A Tabela 1 abaixo apresenta as variáveis que foram estatisticamente significativas para os resultados dos riscos de quedas obtidos nesta pesquisa.

Tabela 1. Variáveis que apresentaram significância estatística para riscos de quedas.

\begin{tabular}{lc}
\hline Variável & $\mathbf{p}$ \\
\hline Idade & 0,0445 \\
Cardiopatias & 0,0169 \\
Distúrbios urinários & 0,0001 \\
Osteoporose & 0,0017 \\
Sequela de AVE & 0,0016 \\
\hline
\end{tabular}

\section{DISCUSSÃO}

Em relação ao risco de quedas os achados foram semelhantes a outros estudos ${ }^{10}$, onde foi investigado 855 idosos independentes, em que $44 \%$ obtiveram um score para baixo risco de queda, $29 \%$ alto risco de quedas e $17 \%$ apresentou risco moderado para queda. E que a queda está intimamente relacionada com o fator idade e com o tipo de doença crônica que o idoso possui.

A pesquisa de Sousa ${ }^{11}$ aponta que mais de 6.000 mulheres, com idade acima de 72 anos, apresentavam incontinência urinaria e dessas, cerca de $25 \%$ tinha um risco maior a sofrer quedas. A incidência de incontinência urinaria em mulheres é de duas a três vezes maiores do que em homens e a relação entre o risco de cair e a presença de incontinência urinária reside basicamente na necessidade de ir ao banheiro varias vezes e sua urgência. A incapacidade de adiamento da micção faz com ele vá muitas vezes ao banheiro e também com o andar acelerado, deixando o idoso mais vulnerável a quedas ${ }^{8,12}$.

A osteoporose, outra variável importante neste estudo, é a causa de fraturas espontâneas de quadril que pode levar queda, ao invés de ser a queda que leva à fratura do quadril. Quedas simples ou outros traumas leves também podem resultar, frequentemente, em fraturas naquelas pessoas que possuem a osteoporose. A autora chama atenção dos gerontólogos em relação aos fatores que aumentam o risco de osteoporose, pois a atuação da equipe de saúde está em inibir o aparecimento desse agravo, tendo em vista que somente o retardado do aparecimento dessa doença é que se previne as quedas causadas por essa desordem ${ }^{13}$. 
Em relação às sequelas de $A V E, a$ pesquisa realizada por Morais $^{14}$ revela que as quedas ocorrem devido à diminuição da capacidade física, mental/cognitiva, reduzindo assim sua capacidade de equilíbrio. Fraquezas, alterações no modo de andar, perda de sensibilidade causada por mudanças neurológicas ou circulatórias, secundárias às comorbidades, estão diretamente ligadas ao risco de cair e necessitam de planos de intervenções sistematicamente construídos por uma equipe de saúde interdisciplinar.

Além de intervenções terapêuticas, há que se destacar a importância da adaptação do meio que o idoso vive no sentido de proporcionar maior segurança e menores riscos. A prevenção de quedas é de responsabilidade de todos e que os centros de convivências, públicos ou privados, que essa população utiliza no seu dia a dia, deve oferecer sessões comunitárias sobre prevenção de quedas e orientações para familiares e cuidadores $^{13}$.

Dentre as várias sugestões para reduzir os riscos de quedas destaca-se a proposta da enfermeira gerontóloga norte americana, Glória Wold $^{13}$, que apresenta uma síntese de intervenções que devem ser realizadas nas comunidades onde os idosos vivem de forma independente, sendo elas: 10 prepare um ambiente seguro: iluminação adequada, mantenha numa mesa próximo à cadeira preferida ou à cama os utensílios mais utilizados, certifique-se que não há tapetes enrugados, piso irregular, fios de eletricidade ou outros objetos que possa fazer a pessoa tropeçar, as poças d'agua devem ser secadas imediatamente, não permita que os idosos subam em bancos ou escadas sem auxílio; 2o permita tempo suficiente para completar uma atividade ou tarefa: a pressa aumenta o risco de quedas e ferimentos porque pode proporcionar tonturas e vertigens por alguns instantes; 3o utilize calçados adequados: estes devem ter solados derrapantes e saltos baixos, com fechos de fácil manipulação e se tiver cadarço, deve certificar-se que não estão frouxos ou desatados, chinelos e sandálias muito abertas são contraindicados; 40 utilize equipamentos auxiliares se necessário: a bengala ou andador amplia a base de sustentação e traz maior segurança, devem ser mantidos à mão para evitar curvar-se ou debruçar-se evitando episódios de tonturas e vertigens momentâneas e 50 peça ajuda sempre que necessário: reconhecer certas necessidades não é sinal de fraqueza, mas sim de bom senso e envelhecimento saudável.

$O$ enfermeiro tem um papel fundamental na elaboração de um plano de cuidado coletivo a partir da discussão das equipes de saúde. Alguns dispositivos podem ser úteis para a elaboração de planos de intervenções interdisciplinares, dentre eles destaca-se o Projeto Terapêutico Singular que consiste em um conjunto de propostas de condutas terapêuticas articuladas, para um sujeito individual ou coletivo, resultado da discussão coletiva de uma equipe interdisciplinar, com apoio matricial e participação ativa do usuárioº .

Entretanto, um dos principais vieses dessa pesquisa foi a quantidade de pessoas avaliadas. Os resultados obtidos e aqui apresentados foram pautados na avaliação de 26 idosos e não é possível afirmar, do ponto de vista estatístico, que esses números se comportariam da mesma forma em populações maiores.

Mesmo assim, os achados potencializam a importância de se realizar intervenções interdisciplinares, principalmente no que tange as ações de enfermagem e fisioterapia junto aos moradores do Programa Vila da Dignidade. Ações interdisciplinares devem vislumbrar a preservação das condições motoras e de equilíbrio do idoso, cercando todos os fatores que estão envolvidos com esses resultados. É necessário que as equipes de saúde compreendam a importância de manter a autonomia dessa população para a realização das atividades básicas e instrumentais da vida diária resultando em melhor qualidade de vida e garantia de condições para morar sozinho, cerne do Programa da Secretaria de Habitação denominado Vila da Dignidade.

\section{CONCLUSÃO}

Este estudo avaliou o risco de quedas em idosos independentes que residem sozinhos. Pequena parte da população estudada possui risco moderado de quedas, e os fatores que foram significativos para esse achado são: idade, presença de cardiopatias, distúrbios urinários, osteoporose e sequela de AVE. A queda, muitas vezes, é para o idoso o gatilho para a síndrome da fragilidade e também pode funcionar como disparador de outros agravos à saúde, como fraturas, internações prolongadas, isolamento social devido ao medo de cair e redução da independência. Apesar de poucos idosos terem apresentado risco de quedas, considera-se 
fundamental que as equipes de saúde do território atuem de forma interdisciplinar a fim traçar medidas que possam prevenir esse evento.

\section{CONFLITOS DE INTERESSE}

Os autores declaram não haver qualquer potencial de conflito de interesse que possa interferir na imparcialidade deste trabalho científico.

\section{REFERÊNCIAS}

1. Vaccari E. Evento queda em idosos hospitalizados. [Dissertação]. Curitiba: Universidade Federal do Paraná, 2013. Curitiba, 2013.

2. Ministério da Saúde (Brasil), Secretaria de Atenção à Saúde, Departamento de Atenção Básica. Envelhecimento e saúde da pessoa idosa. Brasília: Ministério da Saúde; 2006. Acesso em 2 abril 2015. Disponível em: http://bvsms.saude.gov.br/bvs/publicacoes/evel hecimento saude pessoa idosa.pdf

3. Nicolussi AC, Fhon JRS, Santos CAV, Kusumota L, Marques S, Rodrigues RAP. Qualidade de vida em idoso que sofrerão queda: revisão integrativa da literatura. Ciên Saúde Col. 2012;17(3):723-30. Disponível em: http://www.scielo.br/pdf/csc/v17n3/v17n3a19.p $\underline{\mathrm{df}}$

4. São Paulo (Estado). Assembléia Legislativa do Estado de São Paulo. Decreto no. 56.448, de 29 de novembro de 2010. Dá nova redação a dispositivos do Decreto no. 54.285, de 29 de abril de 2009 que autoriza as Secretarias Estaduais da Habitação e de Assistência e Desenvolvimento Social, representando o Estado, a celebrar convênios com a Companhia de Desenvolvimento Habitacional e Urbano do Estado de São Paulo - CDHU e com os Municípios do Estado de São Paulo, visando a implementação do Programa Vila Dignidade. 2010 nov. 29. Acesso em 12 março 2015. Disponível em: http://www.al.sp.gov.br/repositorio/legislacao/d ecreto/2010/decreto-56448-29.11.2010

5. Dias $M$, França $M$, Almeida $B M$, Dias $M K$, Moreno LM, Bittenccourt MA. Mini exame do estado mental: em busca do conhecimento da alteração cognição do idoso na zona oeste do município do Rio de Janeiro. Rev Pesq: cuidado é fundamental online. 2010;2(Supl.):944-947.
6. Paulino CD, Singer JM. Análise de dados categorizados. São Paulo: Edgard Blücher; 2006.

7. Vieira S. Bioestatística: tópicos avançados. 3.ed. Rio de Janeiro: Elsevier; 2010.

8. Bravo Filho J, Kikuchi LE. Geriatria e gerontologia básicas. Rio de Janeiro: Elsevier; 2011.

9. Ministério da Saúde (Brasil), Secretaria de Atenção à Saúde, Departamento de Ações Programáticas e Estratégicas, Atenção à saúde da pessoa idosa e envelhecimento, Ministério da Saúde, Área Técnica Saúde do Idoso. Brasília; 2010. Acesso em 26 out 2015. Disponível em: http://bvsms.saude.gov.br/bvs/publicacoes/aten cao saude pessoa idosa envelhecimento $\mathrm{v} 12 . \mathrm{pdf}$

10. Larêdo PMS. Riscos de quedas em idosos: avaliação da enfermagem através da escala de Tinetti. Rev Hispeci e Lema Online. 2013;4(4):2631.

11. Sousa SLMS. Risco de quedas em mulheres idosas com incontinência urinaria instituicionalizadas [Dissertação]. Escola superior de saúde de Viseu, Instituto Politécnico de Viseu; 2013. Acesso em 22 outubro 2015. Disponível em: http://hdl.handle.net/10400.19/1977

12. Abreu HCA, Reiners AAO, Azevedo RCS, Silva AMC, Abreu DROM. Incontinêencia urinária na predição de quedas em idosos hospitalizados. Rev Esc Enferm USP. 2014;48(5):851-6. DOI: http://dx.doi.org/10.1590/S0080-

623420140000500011

13. Wold G. Enfermagem gerontológica. Rio de Janeiro: Elsevier; 2013.

14. Morais HCC, Holanda GF, Oliveira ARS, Costa AGS, Ximenes CMB, Araujo TL. Identificação do diagnóstico de enfermagem "risco de quedas em idosos com acidente vascular cerebral". Rev Gaúcha Enferm. 2012;33(2):117-24.

Recebido para publicação em 16/08/2016

Revisado em 23/08/2016

Aceito em 13/09/2016 